\title{
Fabrication of Cellulose Aerogel from Sugarcane Bagasse as Drug Delivery Carriers
}

\author{
Suk-Fun Chin, ${ }^{*}$ Fiona Beragai Jimmy and Suh-Cem Pang \\ Department of Chemistry, Faculty of Resource Science and Technology, \\ Universiti Malaysia Sarawak, 94300, Kota Samarahan, Sarawak, Malaysia \\ *Corresponding author: sukfunchin@gmail.com
}

Published online: 25 November 2016

To cite this article: Chin, S. F. et al. (2016). Fabrication of cellulose aerogel from sugarcane bagasse as drug delivery carriers. J. Phys. Sci., 27(3), 159-168, http://dx.doi.org/10.21315/jps2016.27.3.10

To link to this article: http://dx.doi.org/10.21315/jps2016.27.3.10

\begin{abstract}
Currently most of the aerogel-based drug delivery carriers are made from non-biodegradable materials, such as silica. In this study, highly porous cellulose aerogels with Brunauer-Emmett-Teller (BET) surface areas that varied between $22 \mathrm{~m}^{2} \mathrm{~g}^{-1}$ and $525 \mathrm{~m}^{2} \mathrm{~g}^{-1}$ were prepared from a sugarcane bagasse cellulose solution of various concentrations. The potential utility of cellulose aerogels as controlled release carriers was evaluated by loading methylene blue $(M B)$ as a model hydrophilic drug. The MB loading capacity and release kinetic profiles of cellulose aerogels were observed to be substantially influenced by their BET surface areas. Under optimum conditions, a maximum loading capacity of $6.4 \mathrm{mg} \mathrm{MB} \mathrm{mg}^{-1}$ cellulose aerogel was achieved with sustained release of $M B$ from cellulose aerogels at physiological $p H$ over a period of $23 \mathrm{~h}$.
\end{abstract}

Keywords: Cellulose, aerogels, controlled release, drug carriers, sugarcane bagasse

\section{INTRODUCTION}

In recent years, drug delivery carriers have attracted much attention in the healthcare and pharmaceutical industries because they offer the potential to improve therapeutic efficacy, minimise non-specific side effects, prevent drug degradation and enhance the water solubility of drug molecules. ${ }^{1}$ Various types of drug delivery systems, such as nanoparticles, ${ }^{2}$ polymeric micelles, ${ }^{3}$ carbon nanotubes ${ }^{4}$ and nanocapsules ${ }^{5}$ have been extensively studied to achieve optimum drug release kinetics. Aerogels have emerged as promising drug delivery carriers 
due to their high porosity and high specific surface areas, which can provide enhanced drug bioavailability and increased drug loading capacity. ${ }^{6}$ Though silica aerogels have been widely studied as drug delivery carriers, they suffered from drawbacks that include brittleness and non-biodegradability. ${ }^{7}$ Because aerogels are biodegradable, renewable, non-toxic, affordable, have favourable surface functionality and are polysaccharide-based (e.g., starch, alginate, chitosan, pectin, cellulose, etc.) aerogels are more favourable drug delivery carriers. $^{8}$

Starch and alginate aerogels have been studied as controlled release carriers using paracetamol and ibuprofen as model drugs. The drug loading capacity of these polysaccharide-based aerogels was found to be comparable to silica aerogels. ${ }^{9}$ In addition to starch and alginate, cellulose is the most abundant polysaccharide on earth and is another promising precursor material for aerogel synthesis. A bacterial cellulose aerogel loaded with dexpanthenol and L-ascorbic acid has been used for wound dressing applications. The loading capacity and release kinetic profiles of drugs were shown to be affected by the thickness of the aerogel and the solute concentration of the bath solution. ${ }^{10}$ However, aerogels synthesised from bacterial cellulose suffered drawbacks due to shrinking during the drying preparation, which lead to inconsistent pore structures. Recently, Zhao et al. ${ }^{11}$ have prepared a polyethylenimine (PEI) grafted cellulose aerogel from bamboo as a pH-responsive drug delivery carrier. Their work focused on the surface modification of the cellulose aerogel derived from bamboo.

In this study, cellulose aerogels were prepared from cellulose fibres isolated from sugarcane bagasse (SCB). SCB is an agricultural waste that contains high cellulose fibre $(45 \%-55 \%)$ and it is usually burned or used as a low-value product. The utilisation of SCB as a cellulosic source for fabrication of an aerogel-based drug delivery carrier, should result in significant reduction of landfill wastes. Cellulose aerogels with various surface morphology and BET surface areas were prepared by controlling the concentrations of the cellulose solutions. The potential application of these cellulose aerogels as controlledrelease drug delivery carriers were investigated using methylene blue (MB) as the model hydrophilic drug. The effects of the surface morphology and BET surface areas of these cellulose aerogels on their loading capacity and drug release profiles were evaluated under physiological conditions. 


\section{EXPERIMENTAL}

\subsection{Materials}

Sugarcane bagasse was obtained from a local market (Kuching, Sarawak, Malaysia). The sample was first dried under sunlight until it was completely dried and then cut into small pieces $(1$ to $3 \mathrm{~cm})$. The cut bagasse was grounded by using a grinding machine (Model: Quest Scientific; power: $1500 \mathrm{~W}$; speed: $25000 \mathrm{rpm}$ ) to pass a $1.0 \mathrm{~mm}$ size screen of a stainless steel sieve. The grounded bagasse was further dried in an oven for $24 \mathrm{~h}$ at $60^{\circ} \mathrm{C}$. Sodium hydroxide $(\mathrm{NaOH})$, urea, and sodium sulfite were obtained from Merck (Germany); MB, methanol, and absolute ethanol were obtained from $\mathrm{HmbG}$ Chemicals (Germany); thiourea was obtained from Riedel-de Haën (Germany); and sodium chlorite was obtained from J. T. Baker (Sweden). SCB was dried and grinded into powder form. All chemicals were used without further purification. Ultrapure water $\left(\sim 18.2 \mathrm{M} \Omega \mathrm{cm}^{-1}, 25^{\circ} \mathrm{C}\right)$ was obtained from a water purifying system (ELGA, Model Ultra Genetic).

\subsection{Isolation of Cellulose Fibres}

Cellulose fibres were isolated from sugarcane bagasse based using a reported method. ${ }^{12}$ The dried and ground bagasse was first bleached with $0.7 \mathrm{w} / \mathrm{v} \%$ sodium chlorite solution (with a fibre to liquor weight ratio of 1:50) at $\mathrm{pH} 4$ that was adjusted by $5 \mathrm{v} / \mathrm{v} \%$ acetic acid, whereas the mixture was boiled for $5 \mathrm{~h}$ to remove the lignin. The residue was subsequently washed with ultrapure water. The neutral residue was then boiled with $250 \mathrm{ml}$ sodium sulfite $(5 \mathrm{w} / \mathrm{v} \%)$ solution for $5 \mathrm{~h}$, followed by washing with adequate ultrapure water to remove the lignin completely and the hemicelluloses partially. The sample was then boiled with $250 \mathrm{ml} \mathrm{NaOH}(17.5 \mathrm{w} / \mathrm{v} \%)$ for $5 \mathrm{~h}$ to remove the hemicellulose. At the end of the extraction, the insoluble residue (cellulose) was collected through the filtration method and washed thoroughly with ultrapure water until the filtrate was neutral. The sample was kept in a water swollen state during the whole chemical process to avoid generating strong hydrogen bonding among the fibres after the matrix removal.

\subsection{Preparation of Cellulose Aerogels}

Then, $1 \%$ to $5 \% \mathrm{w} / \mathrm{v}$ cellulose solutions were prepared by dissolving cellulose fibres in the aqueous solvent system of sodium hydroxide: thiourea: urea (NTU) $(8: 6.5: 8 \mathrm{w} / \mathrm{v} \%) .{ }^{13}$ The mixture was then dispersed and homogenised with an Ultra-Turrax Disperser, T10 basic (IKA). The suspensions were cooled to $-20^{\circ} \mathrm{C}$ in a freezer for $24 \mathrm{~h}$, and they became a solid frozen mass and were thawed at 
room temperature to obtain a clear cellulosic solution. The mixture was then poured into ethanol (1:2 volume ratio of cellulose solution to ethanol) at a rate of $4 \mathrm{ml}$ per $\mathrm{min}$ and aged at room temperature until the gels were formed (approximately $4 \mathrm{~h}$ ). The gels were then washed with ultrapure water to remove any remaining NTU and soaked in acetone for $1 \mathrm{~h}$ to remove any remaining water. Cellulose aerogel in powder form was obtained when the cellulose wet gel was dried using a supercritical point dryer (Bal-Tec CPD 030). ${ }^{14}$

\subsection{Sample Characterisation}

The surface morphology of the cellulose aerogel was observed using a scanning electron microscope (SEM) (JEOL Model JSM 6390LA). The specific surface areas of the cellulose aerogels were determined using a BET surface area analyser (Quantachrome AutosorbiQ) with the samples degassed at $60^{\circ} \mathrm{C}$ for $4 \mathrm{~h}$.

\subsection{Drug Loading Capacity}

A stock solution of $\mathrm{MB}$ at $500 \mathrm{mM}$ in ethanol was prepared. Then, $50 \mathrm{mg}$ of cellulose aerogels were immersed in $20 \mathrm{ml}$ of $\mathrm{MB}$ solution and equilibrated for 4 $\mathrm{h}$ to achieve maximum loading of MB. The swollen cellulose aerogels were recovered by vacuum filtration and dried at room temperature for $24 \mathrm{~h}$. The concentrations of $\mathrm{MB}$ were determined by measuring the absorbance of the supernatants at a wavelength of $655 \mathrm{~nm}$ with a UV/Vis spectrophotometer (Jasco $\mathrm{V}-630$ ) based on the calibration curve of MB in ethanol. The loading capacity of $\mathrm{MB}$ in cellulose aerogels was calculated based on Equation $1 .{ }^{15}$

$$
\text { Loading capacity }(\mathrm{mg} / \mathrm{mg})=\frac{\mathrm{Mmb}-\mathrm{Msn}}{\mathrm{Ma}}
$$

where $M_{m b}$ is the initial mass of $M B$ in solution, $M_{s n}$ is the mass of $M B$ in the supernatant and $\mathrm{M}_{\mathrm{a}}$ is the mass of the cellulose aerogel. The mass of MB loaded onto the cellulose aerogel was calculated from the difference between the initial concentration of $\mathrm{MB}$ in the solution and the concentration of $\mathrm{MB}$ in the supernatant.

\subsection{Drug Release Kinetic Studies}

Fifty milligrams of MB-loaded cellulose aerogels were dispersed in $20 \mathrm{ml}$ of phosphate buffer solution (PBS) $(\mathrm{pH} 7.4)$ at $37^{\circ} \mathrm{C} \pm 0.5^{\circ} \mathrm{C}$. The supernatants of the centrifuged samples were removed and replaced with the same volume of PBS at predetermined time intervals. The molar concentrations of MB released from cellulose aerogels were determined by measuring the absorbance of the 
supernatants at a wavelength of $655 \mathrm{~nm}$ with a UV/Vis spectrophotometer (Jasco V-630) based on the calibration curve of MB in ethanol. The percentage of MB released from the cellulose aerogel at a given time interval in the supernatant was quantified based on Equation 2:

$$
\text { MB released }(\%)=\frac{[\mathrm{X}] \mathrm{rel}}{[\mathrm{X}] \mathrm{load}} \times 100
$$

where $[\mathrm{X}]_{\text {rel }}$ and $[\mathrm{X}]_{\text {load }}$ are the concentrations of $\mathrm{MB}$ released and loaded, respectively.

\section{RESULTS AND DISCUSSION}

\subsection{Effect of Cellulose Concentration}

Figure 1(a) shows the morphology of a typical cellulose fibre isolated from SCB after the removal of lignin and hemicelluloses. Figure 1(b)-(f) show SEM micrographs of cellulose aerogels synthesised from the cellulose solutions of various concentrations ( 1 to $5 \mathrm{w} / \mathrm{v} \%$ ). All cellulose aerogels were observed to be highly porous in nature and consisted of interwoven nanofibre networks. Aerogels formed from the cellulose solution of higher concentration were observed to be comparatively less porous with a more compact nanofibre structure. This observation concurred with the BET surface areas of the samples, as shown in Table 1. In general, aerogels prepared from a cellulose solution of a higher concentration showed lower BET surface area due to their denser nanofibre network structure. Aerogels with the highest specific surface area of $525 \mathrm{~m}^{2} \mathrm{~g}^{-1}$ were obtained from a cellulose solution of $1 \mathrm{w} / \mathrm{v} \%$, whereas aerogels with the lowest BET surface area of $22 \mathrm{~m}^{2} \mathrm{~g}^{-1}$ were obtained from a cellulose solution of $5 \mathrm{w} / \mathrm{v} \%$. Cellulose aerogels were produced through regeneration (coagulation) by adding the cellulose solutions into a non-solvent (ethanol) at a fixed rate $(4 \mathrm{ml}$ per min). This regeneration process was governed by the nucleation and growth of cellulose molecules during coagulation. Cellulose solution with a higher concentration indicated the presence of higher number of cellulose molecules and therefore increased cellulose/non-solvent molecule interactions that led to the accumulation of cellulose. When the concentration of accumulated cellulose molecules exceeded their limits, solidification occurred, which led to the formation of dense aerogels with little or no porosity. ${ }^{6}$ 


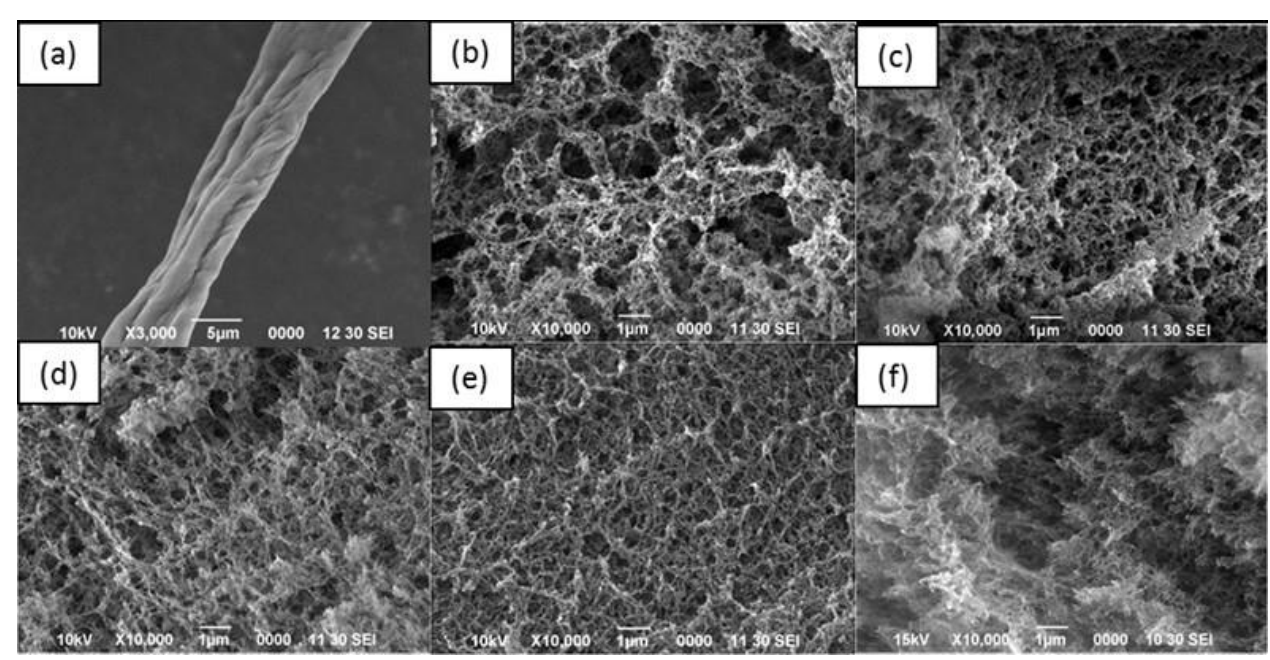

Figure 1: SEM micrographs of (a) cellulose fibres isolated from SCB and cellulose aerogels produced from (b) 1 , (c) 2 , (d) 3 , (e) 4 and (f) 5 w/v \% of SCB cellulose solution.

Table 1: BET surface area from the resultant cellulose aerogels.

\begin{tabular}{cc}
\hline Cellulose aerogel $(\mathrm{w} / \mathrm{v} \%)$ & BET surface area $\left(\mathrm{m}^{2} \mathrm{~g}^{-1}\right)$ \\
\hline 1 & 525 \\
2 & 390 \\
3 & 214 \\
4 & 75 \\
5 & 22 \\
\hline
\end{tabular}

\subsection{Drug Loading Capacity}

As shown in Figure 2, the MB-loading capacity of cellulose aerogel increased with its BET surface area. The highest MB loading capacity of $6.4 \mathrm{mg} / \mathrm{mg}$ was achieved for a cellulose aerogel with the highest BET surface area $\left(525 \mathrm{~m}^{2} \mathrm{~g}^{-1}\right)$, whereas the lowest MB loading capacity of $0.8 \mathrm{mg} / \mathrm{mg}$ was obtained for aerogels with the lowest BET surface area $\left(22 \mathrm{~m}^{2} \mathrm{~g}^{-1}\right)$. MB penetrated into cellulose aerogels and was held in the porous matrices of cellulose aerogels via hydrophilic-hydrophilic interactions. Cellulose aerogels with a higher BET surface area could hold more MB molecules within their porous matrices. In contrast, cellulose aerogels with lower surface area had more compact pore structures that limited the penetration of MB molecules and resulted in a lower loading capacity. ${ }^{16}$ 


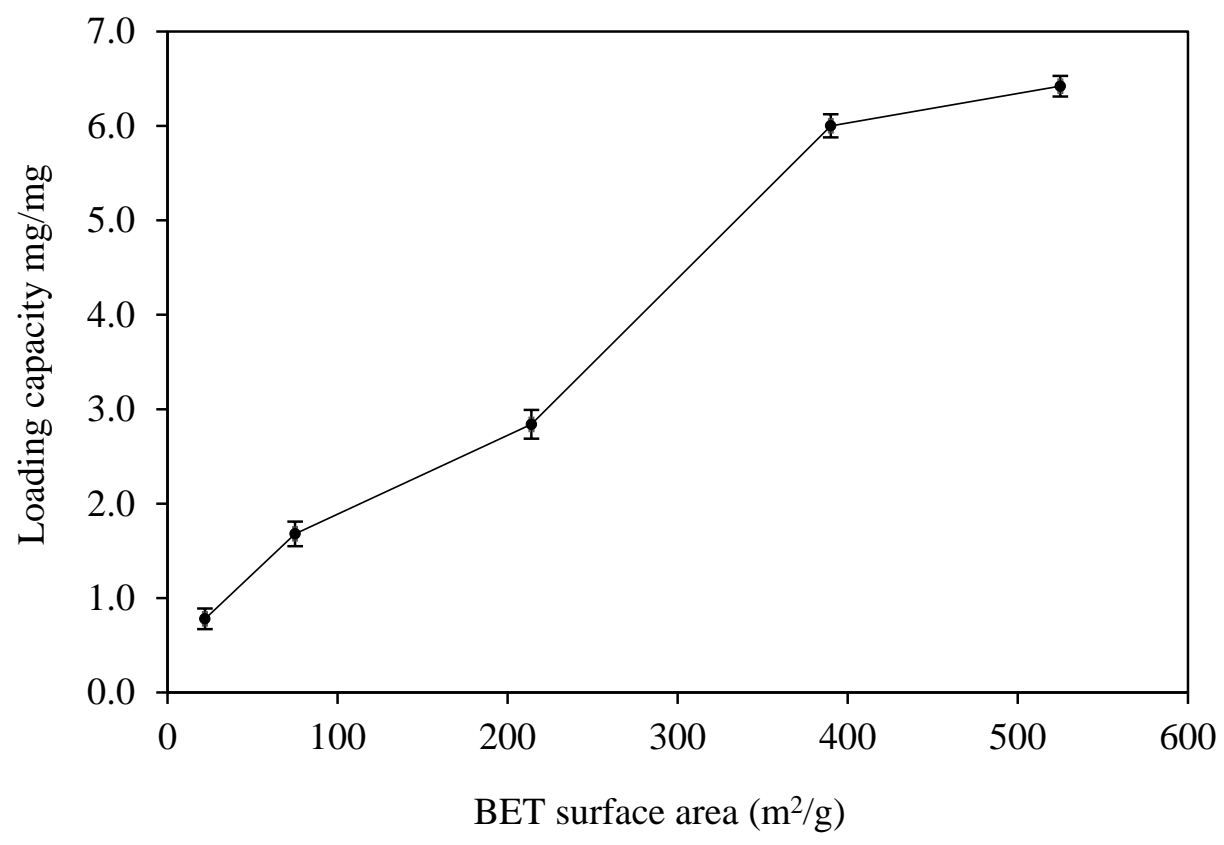

Figure 2: Effect of BET surface area of cellulose aerogel (w/v \%) on loading capacity of MB onto cellulose aerogels.

\subsection{Drug Release Kinetics Profile}

The release kinetics profile of MB from cellulose aerogels with various BET surface areas is shown in Figure 3. Cellulose aerogels with the highest specific surface area of $525 \mathrm{~m}^{2} \mathrm{~g}^{-1}$ exhibited the highest accumulative percentage of MB at $\sim 98 \%$ during the initial $20 \mathrm{~h}$. In contrast, cellulose aerogels with the lowest specific surface area of $22 \mathrm{~m}^{2} \mathrm{~g}^{-1}$ showed a substantially lower cumulative release percentage of $40 \%$ only during the initial $20 \mathrm{~h}$. Cellulose aerogels with a higher BET surface area showed faster overall release rates of MB. Cellulose aerogels with the highest BET surface area $\left(525 \mathrm{~m}^{2} \mathrm{~g}^{-1}\right)$ exhibited the overall fastest rate of MB release and achieved $100 \%$ of MB release within $23 \mathrm{~h}$, which was at a rate of $4.34 \%$ per hour. Yet cellulose aerogels with the lowest BET surface area $\left(22 \mathrm{~m}^{2} \mathrm{~g}^{-1}\right)$ released $100 \%$ of MB within $40 \mathrm{~h}$ at $2.5 \%$ per hour. In other words, it was two times slower than the cellulose aerogel with a BET surface area of $525 \mathrm{~m}^{2} \mathrm{~g}^{-1}$. This release occurred because cellulose aerogels are very hydrophilic. Thus, MB was released from cellulose aerogels through a swelling-controlled mechanism. There were larger contact areas of PBS with aerogel matrices with the highest BET surface areas. As such, PBS could diffuse more readily into cellulose with highly porous structures compared to aerogels with dense and compact structures. A larger contact area of aerogels with PBS solution enabled 
more swelling of the cellulose to occur through absorption of the PBS solution, which in turn caused more MB drug molecules to diffuse out from the swollen matrices and promoted a faster release rate. ${ }^{17}$

\section{CONCLUSION}

In conclusion, cellulose aerogels with a BET surface area ranging from 22 to 525 $\mathrm{m}^{2} \mathrm{~g}^{-1}$ were successfully synthesised from SCB cellulose fibres. The concentration of cellulose was observed to affect the BET surface areas of the resulting cellulose aerogels. The BET surface areas, in turn, had profound impacts on the loading capacity and release kinetics. The loading capacity and release kinetics of the cellulose aerogel could be modulated by tailoring their BET surface areas. Loading of MB onto cellulose aerogels with various BET surface areas afforded a controlled release mechanism with $100 \%$ of the MB being released from cellulose aerogels at physiological $\mathrm{pH} 7.4$ over 23 to $40 \mathrm{~h}$. Cellulose aerogels with the largest BET surface areas $\left(525 \mathrm{~m}^{2} \mathrm{~g}^{-1}\right)$ resulted in the fastest MB release rates $(23 \mathrm{~h})$, whereas cellulose aerogel with the lowest BET surface areas $\left(22 \mathrm{~m}^{2} \mathrm{~g}^{-1}\right)$ exhibited the slowest release rate $(40 \mathrm{~h})$. In addition, these cellulose aerogels were shown to be promising controlled drug release carriers by exhibiting a high-loading capacity $(6.4 \mathrm{mg} / \mathrm{mg})$ and a sustained MB release kinetic profile. The potential utility of cellulose aerogels as efficient and cost-effective drug delivery carriers is therefore a strong possibility.

\section{ACKNOWLEDGEMENTS}

The work was jointly funded by the Committee on Scientific and Technological Corporation/International Foundation of Science (COMSTECH/IFS) under the grant agreement No F/5207-1 and FRGS/STG02(02)/1116/2014(02). The MyBrain15 (MyMaster) programme for graduate scholarship is gratefully acknowledged.

\section{REFERENCES}

1. Alnaief, M. et al. (2012). A novel process for coating of silica aerogel microspheres for controlled drug release applications. Micropor. Mesopor. Mater., 160, 167-173, http://dx.doi.org/10.1016/j.micromeso. 2012.02.009.

2. Pang, S. C. et al. (2015). Fabrication of polysaccharide-based nanoparticles as drug delivery nanocarriers. ECS Trans., 66(37), 15-32, http://dx.doi.org/10.1149/06637.0015ecst. 
3. Miyata, K., Christie, R. J. \& Kataoka, K. (2011). Polymeric micelles for nano-scale drug delivery. React. Funct. Polym., 71(3), 227-234, http://dx.doi.org/10.1016/j.reactfunctpolym.2010.10.009.

4. Karchemski, F. et al. (2012). Carbon nanotubes-liposomes conjugate as a platform for drug delivery into cells. J. Contr. Rel., 160(2), 339-345, http://dx.doi.org/10.1016/j.jconrel.2011.12.037.

5. Mo, J. et al. (2016). Paclitaxel-loaded phosphonated calixarene nanovesicles as a modular drug delivery platform. Sci. Rep., 6, 23489, http://dx.doi.org/10.1038/srep23489.

6. Sehaqui, H., Zhou, Q. \& Berglund, L. A. (2011). High-porosity aerogels of high specific surface area prepared from nanofibrillated cellulose (NFC). Compos. Sci. Technol., 71(13), 1593-1599, http://dx.doi.org/ 10.1016/j.compscitech.2011.07.003.

7. Santamaría, E. et al. (2014). Controlled release of ibuprofen by mesomacroporous silica. J. Solid State Chem., 210(1), 242-250, http://dx.doi.org/10.1016/j.jssc.2013.11.031.

8. Chin, S. F. et al. (2009). Encapsulation and sustained release of curcumin using superparamagnetic silica reservoirs. Chem. Eur. J., 15(23), 5661-5665, http://dx.doi.org/10.1002/chem.200802747.

9. Mehling, T. et al. (2009). Polysaccharide-based aerogels as drug carriers. J. Non-Cryst. Solids, 355, 2472-2479, http://dx.doi.org/10.1016/ j.jnoncrysol.2009.08.038.

10. Haimer, E. et al. (2010). Loading of bacterial cellulose aerogels with bioactive compounds by antisolvent precipitation with supercritical carbon dioxide. Macromol. Symp., 294(2), 64-74, http://dx.doi.org/ 10.1002/masy.201000008.

11. Zhao, J. et al. (2015). Polyethylenimine-grafted cellulose nanofibril aerogels as versatile vehicles for drug delivery. ACS Appl. Mater. Interf., 7(4), 2607-2615, http://dx.doi.org/10.1021/am507601m.

12. Aaltonen, O.\& Jauhiainen, O. (2009). The preparation of lignocellulosic aerogels from ionic liquid solutions. Carbohyd. Polym., 75, 125-129, http://dx.doi.org/10.1016/j.carbpol.2008.07.008.

13. Jin, H., Zha, C. \& Gu, L. (2007). Direct dissolution of cellulose in $\mathrm{NaOH} /$ thiourea/urea aqueous solution. Carbohydr. Res., 342(6), 851-858, http://dx.doi.org/10.1016/j.carres.2006.12.023.

14. Chin, S. F., Romainor, A. N. B. \& Pang, S. C. (2014). Fabrication of hydrophobic and magnetic cellulose aerogel with high oil absorption capacity. Mater. Lett., 115, 241-243, http://dx.doi.org/10.1016/ j.matlet.2013.10.061. 
15. Chin, S. F., Mohd Yazid, S. N. A. \& Pang, S. C. (2014). Preparation and characterization of starch nanoparticles for controlled release of curcumin. Int. J. Polym. Sci., article ID 340121, http://dx.doi.org/ $10.1155 / 2014 / 340121$.

16. Maderuelo, C., Zarzuelo, A. \& Lanao, J. M. (2011). Critical factors in the release of drugs from sustained release hydrophilic matrices. J. Control Rel., 154(1), 2-19.

17. Valo, H. et al. (2011). Immobilization of protein-coated drug nanoparticles in nanofibrillar cellulose matrices-enhanced stability and release. J. Control Rel., 156(3), 390-397. 